\title{
MINIMAL DARBOUX TRANSFORMATIONS
}

\author{
U. HERTRICH-JEROMIN AND A. HONDA
}

\begin{abstract}
We derive a permutability theorem for the Christoffel, Goursat and Darboux transformations of isothermic surfaces. As a consequence we obtain a simple proof of a relation between Darboux pairs of minimal surfaces in Euclidean space, curved flats in the 2-sphere and flat fronts in hyperbolic space.
\end{abstract}

\section{INTRODUCTION}

Recently, Martínez-Roitman-Tenenblat 13 obtained a formula for the Weierstrass data of minimal surfaces in Euclidean 3 -space $\boldsymbol{R}^{3}$ that are related by a Ribaucour transformation [13, Cor 3.4], which was used to analyze the asymptotic behavior and the umbilic points of minimal surfaces obtained by Ribaucour transformations. To derive this formula, the authors use the cyclographic projection, a correspondence between (oriented) spheres in $\boldsymbol{R}^{3}$ and the Minkowski 4-space $\boldsymbol{R}_{1}^{4}$. In this way, they obtain a relation between flat fronts in hyperbolic 3-space and minimal surface in $\boldsymbol{R}^{3}$.

We aim to provide a simpler, virtually computation-free account of these relations, based on a permutability theorem for isothermic surfaces.

In [9, see also [11, Sect 5.3], the first author extended the Goursat transformation - classically known for minimal surfaces, see 8 - to the class of isothermic surfaces and argued that the classical Enneper-Weierstrass representation can be regarded as a special case of this "extended" Goursat transformation, see [11, §5.3.12]. As our main Theorem 2.1 we derive a new permutability theorem for the transformations of isothermic surfaces, involving this Goursat transformation.

The aforementioned relation between the Weierstrass data of minimal surfaces related by a Darboux tranformation is then obtained as a degenerate case of our permutability theorem, see Corollary 3.1 .

For the relation to flat fronts in hyperbolic space we draw on the relation between Darboux pairs of isothermic surfaces and curved flats in the space of point pairs in the conformal 3-sphere, see [2] or 11, §3.3.2]. By the well known Bianchi permutability theorem for the Christoffel and Darboux transformations, see [1, §5], a Darboux pair of minimal surfaces yields a curved flat in the 2-sphere, thus a pair

Date: February 23, 2016.

2010 Mathematics Subject Classification. Primary 53A10, 37K35; Secondary 53C42, 53A30, $37 \mathrm{~K} 25,34 \mathrm{M} 45$.

Key words and phrases. Minimal surface, Darboux transformation, Christoffel transformation, Goursat transformation, Bianchi permutability, Riccati equation, flat front, curved flat, hyperbolic geometry.

This work has been partially supported by the Austrian Science Fund (FWF) and the Japan Society for the Promotion of Science (JSPS) through the FWF/JSPS Joint Project grant I1671N26 "Transformations and Singularities". 
of hyperbolic Gauss maps of a parallel family of flat fronts in hyperbolic space, cf [13, Thms $3.2 \& 3.3$ ], or [4, Sect 3].

\section{Preliminaries}

Our main focus will be on minimal surfaces in the Euclidean space $\boldsymbol{R}^{3}$, regarded as special isothermic surfaces, that is, surfaces that admit (local) conformal curvature line coordinates around every non-umbilic point. Thus given a conformal parametrization

$$
f: M^{2} \rightarrow \boldsymbol{R}^{3}
$$

of an isothermic surface over a Riemann surface $M^{2}$, it will often be useful employ conformal curvature line parameters $(u, v): M^{2} \rightarrow \boldsymbol{R}^{2}$, where the first and second fundamental forms of the surface become particularly simple:

$$
\langle d f, d f\rangle=E\left(d u^{2}+d v^{2}\right) \text { and }\langle d f, d n\rangle=-E\left(\kappa_{1} d u^{2}+\kappa_{2} d v^{2}\right) .
$$

Note that we do not exclude umbilics, in fact, we will need to consider the unit sphere as an isothermic surface, with any conformal coordinates providing a conformal "curvature line parametrization".

To facilitate our analysis we consider Euclidean 3-space

$$
\boldsymbol{R}^{3} \cong \operatorname{Im} \boldsymbol{H}=\left\{x_{1} i+x_{2} j+x_{3} k ; x_{1}, x_{2}, x_{3} \in \boldsymbol{R}\right\}
$$

as the set of imaginary quaternions, thereby obtaining the Hamiltonian product

$$
x y=-\langle x, y\rangle+x \times y,
$$

of vectors as an additional algebraic structure. However, we remark that we can replace the Hamiltonian product by the Clifford product with only minor adjustments, see [3] or [11, Chapter 8]; see also Remark 2.2.

A key argument of our analysis will be the conformal invariance of the notion of an isothermic surface: in particular, if $f: M^{2} \rightarrow \boldsymbol{R}^{3}$ is isothermic then so is any of its Möbius transforms

$$
\mu \circ f: M^{2} \rightarrow \boldsymbol{R}^{3}, \quad \mu(x)=(a x+b)(c x+d)^{-1} .
$$

1.1. Christoffel's transformation. Given an isothermic surface $f: M^{2} \rightarrow \boldsymbol{R}^{3}$ there is locally a second surface $f^{*}: M^{2} \rightarrow \boldsymbol{R}^{3}$ such that (cf. [5] and [11, §5.2.1]):

- $f$ and $f^{*}$ have parallel tangent planes at corresponding points, $d f\left(T_{p} M^{2}\right)=$ $d f^{*}\left(T_{p} M^{2}\right)$ for each $p \in M^{2}$

- the shape operators $A, A^{*}$ of $f$ and $f^{*}$ commute (i.e., the curvature directions coincide in $T_{p} M^{2}$; cf. [11, Lemma 3.1.6]), and $\operatorname{det} A^{*} \operatorname{det} A \leq 0$;

- $f$ and $f^{*}$ induce conformally equivalent metrics.

Such a surface $C(f):=f^{*}$ is called a Christoffel transform or Christoffel dual of $f$. Away from umbilics, a Christoffel dual is unique up to homothety: given conformal curvature line coordinates $(u, v)$ for $f$, a Christoffel dual $f^{*}$ is obtained by integrating Christoffel's formula

$$
d f^{*}=\frac{2}{E\left(\kappa_{1}-\kappa_{2}\right)}(d n+H d f)=-\frac{1}{E}\left(f_{u} d u-f_{v} d v\right) .
$$

Alternatively, $f^{*}$ may be characterized by the fact that

$$
q:=d f d f^{*}=\left(d u^{2}-d v^{2}\right)+2 n d u d v
$$


defines a holomorphic quadratic differential when identifying the trivial normal bundle of $f$ in $\boldsymbol{H}$ with $\boldsymbol{C}$ via $n \mapsto i$. This demonstrates the symmetry of the Christoffel duality, that is, the fact that it can reasonably be called a "duality", cf [10, Thm 4] and [11, §5.2.6]:

$$
f^{* *}=f
$$

As the Gauss map $n: M^{2} \rightarrow S^{2}$ of a minimal surface $f: M^{2} \rightarrow \boldsymbol{R}^{3}$ is conformal, it yields a Christoffel dual of $f$ as an isothermic surface, $f^{*}=n$. In fact, minimal surfaces in Euclidean space can be characterized as isothermic surfaces whose Gauss map yields a Chrstoffel dual, see [10, [11, §5.2.10]. In order to "reverse engineer" a minimal surface $f=n^{*}$ from its Gauss map $n$ via Christoffel duality, more input data is required: prescribing a holomorphic quadratic differential $q$ on $M^{2}$ fixes a "curvature line net" on the spherical patch $n\left(M^{2}\right) \subset S^{2}$, thus fixes a notion of conformal "curvature line parameters" that allow to use Christoffel's formula to uniquely determine a Christoffel dual

$$
f=n^{*}=-\int \frac{n_{u} d u-n_{v} d v}{E}
$$

from $n$. Alternatively, other holomorphic data can be prescribed on the Riemann surface $M^{2}$ to obtain the holomorphic Hopf differential of the minimal surface $f$ and hence the minimal surface via Christoffel's formula, cf. [11, §5.2.11 and §5.3.12].

1.2. Goursat's transformation. Describing a minimal surface $f=\operatorname{Re} \Psi$ as the real part of a holomorphic null curve $\Psi: M^{2} \rightarrow C^{3}$ it is clear that any orthogonal transformation $A \in O(3, \boldsymbol{C})$ yields a new minimal surface via

$$
\tilde{f}:=\operatorname{Re} \tilde{\Psi} \text { with } \tilde{\Psi}:=A \Psi,
$$

a Goursat transform of $f$, cf. [8], [14, §83]. It is well know that the effect of such a Goursat transform on the Gauss map is a Möbius transformation, thereby suggesting a extension of the notion to isothermic surfaces, see [9] or [11, Def 5.3.3]: given an isothermic surface $f$ and a Möbius transformation $\mu$ of $\boldsymbol{R}^{3} \cup\{\infty\}$, the (locally defined) surface

$$
\tilde{f}:=\left(\mu \circ f^{*}\right)^{*}
$$

will be called a Goursat transform of $f$. We also write $G_{\mu}(f):=\tilde{f}$. In particular, for $\mu(x)=(x-z)^{-1}$ with $z \in \operatorname{Im} \boldsymbol{H}$, we have (cf. [11, Lemma 5.3.10])

$$
d \tilde{f}=-\left(f^{*}-z\right) d f\left(f^{*}-z\right) .
$$

1.3. Darboux's transformation. A pair of surfaces $f, \hat{f}: M^{2} \rightarrow \boldsymbol{R}^{3}$ is called a Ribaucour pair if

- for each $p \in M^{2}$, there is a 2-sphere $s(p)$ that has first order contact with both $f$ at $f(p)$ and $\hat{f}$ at $\hat{f}(p)$; at $p$, and

- the shape operators of $f$ and $\hat{f}$ commute;

and a Ribaucour pair is called a Darboux pair (cf. [6]) if, additionally,

- their induced metrics are conformally equivalent.

Given an isothermic surface $f: M^{2} \rightarrow \boldsymbol{R}^{3}$ and a parameter $t \in \boldsymbol{R} \backslash\{0\}$, a Darboux transform $\hat{f}$ of $f$ is obtained by integrating the following completely integrable Riccati equation (cf. [10] or [11, §5.4.8f])

$$
d \hat{f}=t(\hat{f}-f) d f^{*}(\hat{f}-f) .
$$


As a consequence, an isothermic surface admits, locally, a $(1+3)$-parameter family of Darboux transforms, depending on the "spectral parameter" $t \in \boldsymbol{R}$ and the choice of an initial point of the transform. We note that the Darboux transformation is invariant under Möbius transformations, that is, if $\mu$ is a Möbius transformation then

$$
\mu\left(D_{t}(f)\right)=D_{t}(\mu(f))
$$

Note that the Riccati equation (1.2) breaks the symmetry of the definition of the Darboux transformation: as the Darboux transformation is involutive, $\hat{f}=D_{t}(f)$ iff $f=D_{t}(\hat{f})$, we also have a corresponding Riccati equation

$$
d f=t(\hat{f}-f) d \hat{f}^{*}(\hat{f}-f) .
$$

1.4. Bianchi's permutability theorem. The Christoffel and Darboux transformations commute, that is, the following diagram commutes, see [1, §5] or [11, Thm 5.6.3]:

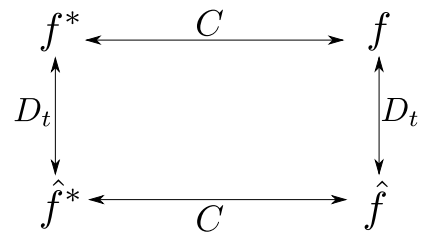

More precisely, given a Christoffel dual $f^{*}$ and a Darboux transform $\hat{f}=D_{t}(f)$ of an isothermic surface $f: M^{2} \rightarrow \boldsymbol{R}^{3}=\operatorname{Im} \boldsymbol{H}$, the surface

$$
\hat{f}^{*}:=f^{*}+\frac{1}{t}(\hat{f}-f)^{-1}
$$

is a Christoffel dual of $\hat{f}$ and a $D_{t}$-transform of $f^{*}$ at the same time.

Note that this permutability scheme occurs as a limiting case of the commonly known Bianchi permutability scheme of [1, §3] (cf. [11, §5.6.6]) for the Darboux transformations when one of the spectral parameters vanishes.

\section{Permutability theorem}

We are now prepared to state and prove our main permutability theorem for the Christoffel, Goursat and Darboux transformations:

Theorem 2.1. Given a Darboux transform $\hat{f}=D_{t}(f)$ and a Goursat transform $\tilde{f}=G_{\mu}(f)$ of an isothermic surface $f: M^{2} \rightarrow \boldsymbol{R}^{3}$, the surface

$$
G_{\mu}(\hat{f}):=G_{\mu}(f)+\frac{1}{t}\left(\mu \circ \hat{f}^{*}-\mu \circ f^{*}\right)^{-1}
$$

is simultaneously a Goursat transform of $\hat{f}$ and a $D_{t}$-transform of $\tilde{f}$. Moreover the following diagram commutes: 


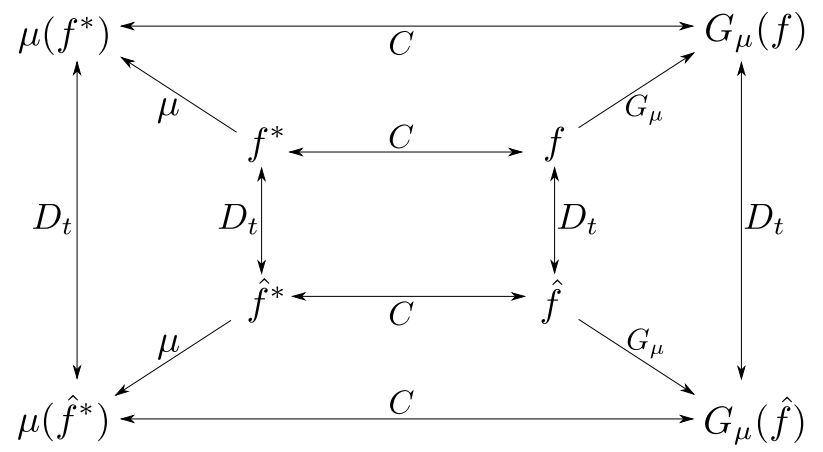

Proof. Let $f$ be isothermic and $G_{\mu}(f)$ and $D_{t}(f)$ a Goursat resp. Darboux transform of $f$. The Goursat transform $G_{\mu}(f)$ is given by intertwining the Christoffel duality with a Möbius transformation,

$$
G_{\mu}(f)=\left(\mu \circ f^{*}\right)^{*} .
$$

Thus the upper quadrilateral of our diagram reflects the definition of the Goursat transformation. Then, by Bianchi's permutability theorem, (1.5) can serve to complete the inner quadrilateral of the diagram,

$$
\hat{f}^{*}=f^{*}+\frac{1}{t}(\hat{f}-f)^{-1} .
$$

Now the Möbius invariance of $D_{t}$ (cf. (1.3) ) yields the left quadrilateral in the diagram,

$$
D_{t}\left(\mu\left(f^{*}\right)\right)=\mu\left(D_{t}\left(f^{*}\right)\right)=\mu\left(\hat{f}^{*}\right),
$$

and another application of Bianchi's permutability theorem serves to obtain

$$
f^{\dagger}:=G_{\mu}(f)+\frac{1}{t}\left(\mu \circ \hat{f}^{*}-\mu \circ f^{*}\right)^{-1}
$$

as a Darboux transform of $G_{\mu}(f)$ and a Christoffel dual of $\mu \circ \hat{f}^{*}$ at the same time,

$$
f^{\dagger}=D_{t}\left(G_{\mu}(h)\right)=\left(\mu \circ \hat{f}^{*}\right)^{*}=G_{\mu}(\hat{f}),
$$

where the last equality is due to the definition of the Goursat transformation, again.

Remark 2.2. Although we used the Hamiltonian product on $\boldsymbol{R}^{3}=\operatorname{Im} \boldsymbol{H}$ thought of as the space of imaginary quaternions, all considerations can equally well be formulated using the Clifford product on $\boldsymbol{R}^{3}$. This fact hinges on how the symmetric parts of both, the Hamiltonian and Clifford products, are related to the inner product of $\boldsymbol{R}^{3}$, so that the geometric content of equations such as the Riccati equation (1.2) can equivalently be expressed in terms of either product, cf. [3. Sect 2 ] or [11, Chap 8]. However, the Clifford product can be employed in $\boldsymbol{R}^{n}$, hence Theorem 2.1 holds in arbitrary codimension.

\section{Weierstrass data of minimal Darboux transformations}

Now we focus on the particular Möbius transformation,

$$
\iota: \boldsymbol{R}^{3} \cup\{\infty\} \rightarrow \boldsymbol{R}^{3} \cup\{\infty\}, x \mapsto \iota(x):=-i-2(i+x)^{-1},
$$


which yields the stereographic projection and its inverse when restricted to the 2-sphere $S^{2} \subset \boldsymbol{R}^{3}$ and $\boldsymbol{C}=\{i\}^{\perp} \subset \boldsymbol{R}^{3}$, respectively:

$$
\left.\iota\right|_{S^{2}}=\sigma \text { and }\left.\iota\right|_{\boldsymbol{C} j}=\sigma^{-1} \text {. }
$$

From two holomorphic (or meromorphic) functions $g, h: M^{2} \rightarrow \boldsymbol{C}$ on the Riemann surface $M^{2}$ we obtain a degenerate Christoffel pair

$$
h j, \quad(h j)^{*}=-j g,
$$

cf. [11, §5.2.11]. Following [11, §5.3.12] we consider the Goursat transformation $f=G_{\iota}(h j)$ that is obtained as a Christoffel transform of the (holomorphic) Gauss map

$$
n=\mu \circ(h j)^{*}=\frac{1}{1+|g|^{2}}\left(\left(1-|g|^{2}\right) i-2 j g\right): M^{2} \rightarrow S^{2},
$$

by integrating

$$
d f=2 \operatorname{Re}(g \omega) i+\operatorname{Re}\left(\left(1-g^{2}\right) \omega\right) j+\operatorname{Im}\left(\left(1+g^{2}\right) \omega\right) k
$$

with $\omega:=\frac{1}{2} d h$, as obtained from (1.1). This identifies the Weierstrass representation for minimal surfaces as a special case of the Goursat transformation for isothermic surfaces, as annunciated above.

Thus we consider the Weierstrass data of the minimal surface $f$ to be one of the following equivalent sets of data:

- the pair $(g, h)$ of the meromorphic resp. holomorphic functions $g$ and $h$;

- the pair $(g, \omega)$ of the meromorphic function $g$ and a suitable holomorphic differential $\omega$; or

- the pair $(g, q)$ of the meromorphic function $g$ and a holomorphic quadratic differential $q$ (i.e., the "polarization" of $M^{2}$ ).

Here the second items of these data sets are related by the equations

$$
2 \omega d g=d h d g=q .
$$

Note that, for a minimal surface $f$ with its Gauss map $n$ as a Christoffel transform, our holomorphic quadratic differential is related to its Hopf differential by

$$
q=d h d g \simeq d f d f^{*}=2\left(\operatorname{Re}\left\langle f_{z z}, n\right\rangle d z^{2}+n \operatorname{Im}\left\langle f_{z z}, n\right\rangle d z^{2}\right) .
$$

3.1. Application of Theorem 2.1. As an application of Theorem 2.1 we can now derive a formula that relates the Weierstrass data of the surfaces of a minimal Darboux pair, of [13, Cor 3.4]:

Corollary 3.1. Let $M^{2}$ be a Riemann surface and $f, \hat{f}: M^{2} \rightarrow \boldsymbol{R}^{3}$ non-flat conformal minimal immersions, given by Weierstrass data $(g, q)$ and $(\hat{g}, \hat{q})$, respectively. Then $(f, \hat{f})$ is, up to translation, a Darboux pair if and only if there is $t \in \boldsymbol{R} \backslash\{0\}$ so that

$$
d g d \hat{g}=t(\hat{g}-g)^{2} q \text { and } \hat{q}=q .
$$

Here we consider $q$ and $\hat{q}$ as $\boldsymbol{C}$-valued holomorphic quadratic differentials.

Note that, with $\omega:=\frac{q}{2 d g}$, the first equation of (3.3) yields the Riccati equation

$$
d \hat{g}=2 t(\hat{g}-g)^{2} \omega
$$


of [13. (3.11)], while the transformation formula [13, (3.12)] for the Weierstrass data is obtained using $\hat{q}=q$ :

$$
\hat{\omega}=\frac{\hat{q}}{2 d \hat{g}}=\frac{d g}{2 t(\hat{g}-g)^{2}} .
$$

A lemma will be helpful to prove the theorem, cf [10, (58)] or [11, §5.4.15]:

Lemma 3.2. Let $f: M^{2} \rightarrow \boldsymbol{R}^{3}$ be isothermic, and let $f^{*}$ and $\hat{f}=D_{t}(f)$ denote a Christoffel and a Darboux transform of $f$, respectively. Then

$$
0=\hat{H}|\hat{f}-f|^{2}-2\langle\hat{f}-f, n\rangle+\frac{1}{t} H^{*},
$$

where $n$ denotes a Gauss map of $f$, and the Gauss maps $n^{*}$ and $\hat{n}$ are chosen so that the holomorphic quadratic differentials $\hat{q} \simeq q^{*} \simeq q$.

Proof. Given a Gauss map $n$ of $f$, there is a consistent choice of Gauss maps

$$
n^{*}:=-n \text { and } \hat{n}:=-(\hat{f}-f)^{-1} n(\hat{f}-f)
$$

so that $\hat{q} \simeq q^{*} \simeq q$ when identifying $\hat{n} \simeq n^{*} \simeq n$ as imaginary units of the normal bundles of $\hat{f}, f^{*}$ and $f$ in $\boldsymbol{H}$, as complex rank 1 bundles: using $f^{* *}=f$,

$$
q^{*}=d f^{*} d f=\overline{d f d f^{*}}=\bar{q}, \text { hence } q^{*} \simeq q ;
$$

and using the Riccati equations and Bianchi's permutability for the Christoffel and Darboux transformations,

$$
d \hat{f}=t(\hat{f}-f) d f^{*}(\hat{f}-f) \text { and } \hat{f}^{*}=f^{*}+\frac{1}{t}(\hat{f}-f)^{-1},
$$

we deduce that

$$
\hat{q}=d \hat{f} d \hat{f}^{*}=(\hat{f}-f) d f^{*} d f(\hat{f}-f)^{-1}=(\hat{f}-f) \bar{q}(\hat{f}-f)^{-1}, \text { hence } \hat{q} \simeq q .
$$

Then direct computation yields with $n^{*}=-n$

$$
d \hat{n}=(\hat{f}-f)^{-1}\left\{d n^{*}+2\langle\hat{f}-f, n\rangle\left(t d f^{*}-\frac{1}{|\hat{f}-f|^{2}} d f\right)\right\}(\hat{f}-f),
$$

hence the second fundamental form of $\hat{f}$ with $\hat{n}$ as a normal field computes to

$$
\operatorname{Re} d \hat{f} d \hat{n}=-t|\hat{f}-f|^{2}\left\{\operatorname{Re} d f^{*} d n^{*}-2 t\langle\hat{f}-f, n\rangle\left\{t\left|d f^{*}\right|^{2}+\frac{1}{|\hat{f}-f|^{2}} \operatorname{Re} q^{*}\right\}\right\}
$$

and taking trace with respect to $|d \hat{f}|^{2}=t^{2}|\hat{f}-f|^{4}\left|d f^{*}\right|^{2}$ yields the claim since $q^{*}$ is a quadratic differential so that $\operatorname{Re} q^{*}$ is trace free.

Now Corollary 3.1 follows directly from our Permutability Theorem 2.1

Proof. First assume that (3.3) holds for some $t \in \boldsymbol{R}$, that is, with $d(-j g)^{*}=\frac{q}{d g} j$ we have

$$
d(-j \hat{g})=t(-j \hat{g}+j g) d(-j g)^{*}(-j \hat{g}+j g)
$$

showing that $-j \hat{g}=D_{t}(-j g)$ : hence, wheeling out our Permutability Theorem 2.1 we learn that $f$ and $\hat{f}$ form a Darboux pair when appropriately positioned in $\boldsymbol{R}^{3}$.

To see the converse we employ Lemma 3.2 with $H^{*}=1$ and $\hat{H}=0$ this yields

$$
\hat{f}^{*}=f^{*}+\frac{1}{t}(\hat{f}-f)^{-1}=n+2\langle\hat{f}-f, n\rangle(\hat{f}-f)^{-1}=\hat{n},
$$

Hence, unleashing our Permutability Theorem 2.1 gaain we obtain the result, as above. 
As a direct consequence of (2.1) from Thm 2.1 and Cor 3.1 we obtain:

Corollary 3.3. Let $f, \hat{f}: M^{2} \rightarrow \boldsymbol{R}^{3}$ form a minimal Darboux pair, with Gauss maps $n$ resp $\hat{n}$. Then

$$
\hat{f}=f+\frac{1}{t}(\hat{n}-n)^{-1} \text {. }
$$

3.2. Curved flats and flat fronts. By Corollary 3.1 resp [13, Cor 3.4], the Gauss maps $n$ and $\hat{n}$ of a minimal Darboux pair $(f, \hat{f})$ in Euclidean space form a (degenerate) Darboux pair themselves, hence a curved flat in the space of point pairs in the 2-sphere, see [7] or [11, $\S 3.3 .2$ or $\S 5.5 .20]$. Consequently, interpreting the interior of the 2-sphere as a Poincaré ball model of hyperbolic space, $(n, \hat{n})$ qualifies as the pair of hyperbolic Gauss maps of a parallel family of flat fronts, see 12. More precisely, linearization of of the Riccati equations (3.3) for $\hat{g}$ reads

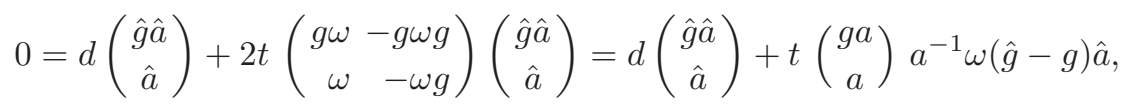

where $d \hat{a}+2 t \omega(\hat{g}-g) \hat{a}=0$, and similary for $d g$. Thus we obtain the curved flat system

$$
d\left(\begin{array}{cc}
g a & \hat{g} \hat{a} \\
a & \hat{a}
\end{array}\right)+t\left(\begin{array}{cc}
g a & \hat{g} \hat{a} \\
a & \hat{a}
\end{array}\right)\left(\begin{array}{cc}
0 & a^{-1} \omega(\hat{g}-g) \hat{a} \\
\hat{a}^{-1} \hat{\omega}(g-\hat{g}) a & 0
\end{array}\right)=0
$$

of the Weierstrass type representation for flat fronts in hyperbolic space.

Thus we recover the relation between minimal Darboux pairs in Euclidean space and (parallel families of) flat fronts in hyperbolic space of [13, Thms $3.2 \& 3.3$ ], see also [4, Sect 3]:

Corollary 3.4. Any minimal Darboux pair $(f, \hat{f})$, with $(n, \hat{n})$ as its Darboux pair of Gauss maps, determines a parallel family of flat fronts in hyperbolic space, with hyperbolic Gauss maps $n$ and $\hat{n}$.

Conversely, the hyperbolic Gauss maps of a flat front in hyperbolic space form a curved flat in the 2-sphere, hence determine a minimal Darboux pair in Euclidean space.

Note that the above linearization of the Riccati equations can equally be performed for maps into $\boldsymbol{R}^{3}$ or $\boldsymbol{R}^{n}$ by using quaternions or an appropriate Clifford algebra, see [11, §5.4.1 and $\S 8.7 .2]$ or [3, Sect 3.2].

Acknowledgements. The second author expresses his gratitude to the members of the Institute of Discrete Mathematics and Geometry at TU Wien for their hospitality during his stay in 2015/16.

\section{REFERENCES}

[1] L. Bianchi, Il teorema di permutabilità per le trasformazioni di Darboux delle superficie isoterme, Rom. Acc. L. Rend. 13 (1904), 359-367.

[2] F.E. Burstall, U. Hertrich-Jeromin, F. Pedit, U. Pinkall, Curved flats and isothermic surfaces, Math. Z. 225 (1997) 199-209

[3] F.E. Burstall, Isothermic surfaces: conformal geometry, Clifford algebras and integrable systems, Integrable systems, geometry, and topology, AMS/IP Stud. Adv. Math., vol. 36, Amer. Math. Soc., Providence, RI, 2006, pp. 1-82.

[4] F.E. Burstall, I. Hertrich-Jeromin, W. Rossman: Lie geometry of flat fronts in hyperbolic space, C. R. 348 (2010) 661-664

[5] E. Christoffel, Ueber einige allgemeine Eigenschaften der Minimumsflächen, J. Reine Angew. Math. 67 (1867), 218-228. 
[6] G. Darboux, Sur les surfaces isothermiques, C.R. Acad. Sci. Paris 128 (1899), 1299-1305, 1538 .

[7] D. Ferus and F. Pedit, Curved flats in symmetric spaces, Manuscripta Math. 91 (1996), 445-454.

[8] E. Goursat, Sur un mode de transformation des surfaces minima, Acta Math. 11 (1887), 135-186, 257-264.

[9] U. Hertrich-Jeromin, Supplement on curved flats in the space of point pairs and isothermic surfaces: a quaternionic calculus, Doc. Math. 2 (1997), 335-350.

[10] U. Hertrich-Jeromin and F. Pedit, Remarks on the Darboux transform of isothermic surfaces, Doc. Math. 2 (1997), 313-333.

[11] U. Hertrich-Jeromin, Introduction to Möbius differential geometry, London Mathematical Society Lecture Note Series, vol. 300, Cambridge University Press, Cambridge, 2003.

[12] M. Kokubu, M. Umehara and K. Yamada, Flat fronts in hyperbolic 3-space, Pacific J. Math. 216 (2004), 149-175.

[13] A. Martínez, P. Roitman, and K. Tenenblat, A connection between flat fronts in hyperbolic space and minimal surfaces in euclidean space, Ann. Global Anal. Geom. 48 (2015), 233-254.

[14] J.C.C. Nitsche, Lectures on minimal surfaces. Vol. 1, Cambridge University Press, Cambridge, 1989.

(Udo Hertrich-Jeromin) Vienna University of Technology, Wiedner Hauptstrasse 810/104, A-1040 Vienna, Austria

E-mail address: udo.hertrich-jeromin@tuwien.ac.at

(Atsufumi Honda) National Institute of Technology, Miyakonojo College, Yoshio, MiYAKONOJO 885-8567, JAPAN

E-mail address: atsufumi@cc.miyakonojo-nct.ac.jp 